\title{
Relação entre a Classificação Internacional de Funcionalidade, Incapacidade e Saúde (CIF) e a limitação de atividades e restrição à participação de indivíduos com hanseníase
}

\section{Relationship between the International Classification of Functioning, Disability and Health (ICF) and the limitation of activities and restriction of participation for individuals with leprosy}

\author{
Elcemir Galvão e Silva de Moura¹, Ana Paula Monteiro de Araújo², \\ Maria Clara Raiol da Silva², Biatriz Araújo Cardoso ${ }^{1,3,4}$, \\ Michelle Castro da Silva Holanda ${ }^{1,2}$, Adélia Oliveira da Conceição ${ }^{1}$, \\ George Alberto da Silva Dias²
}

\begin{abstract}
Resumo
Introdução: Hanseníase é uma doença infecciosa que pode levar à incapacidade física. A escala SALSA e a Classificação Internacional de Funcionalidade, Incapacidade e Saúde (CIF) podem ser úteis no manejo clínico de pacientes com essa doença. Assim, o objetivo foi relacionar a CIF com a limitação de atividade e restrição à participação de indivíduos com hanseníase, utilizando a escala SALSA. Metodologia: Tratou-se de um estudo observacional transversal analítico e descritivo, com a utilização da escala SALSA, relacionado aos domínios mobilidade, autocuidado, trabalho e destreza. Após a entrevista realizada com os pacientes, os domínios foram codificados pela CIF segundo os códigos relacionados à atividade e participação. Resultados: $A$ amostra foi composta por 30 indivíduos, com média de idade de 38,4, sendo a maioria do gênero masculino. A média do escore da escala SALSA foi de 36,9 pontos, apresentando leve limitação de atividades. Os domínios da escala SALSA e o uso dos códigos da CIF mostraram dificuldade leve a moderada em sua grande maioria, com resultados significantes $(p \leq 0,05)$. $O$ único domínio que apresentou tarefa com dificuldade completa foi o de mobilidade. Conclusão: A escala SALSA mostrou leve limitação de atividade em pacientes com hanseníase, porém, quando relacionado à CIF, apresentou limitações maiores, principalmente no domínio mobilidade.
\end{abstract}

Palavras-chave: hanseníase; Classificação Internacional de Funcionalidade, incapacidade e saúde; fisioterapia.

'Universidade da Amazônia (UNAMA) - Belém (PA), Brasil.

2Universidade do Estado do Pará (UEPA) - Belém (PA), Brasil.

${ }_{3}^{3}$ Programa de Pós-graduação em Medicina Tropical, Instituto Oswaldo Cruz (IOC), Fundação Oswaldo Cruz (FIOCRUZ) - Rio de Janeiro (RJ), Brasil.

4Faculdade Metropolitana da Amazônia (FAMAZ) - Belém (PA), Brasil.

Trabalho realizado na Universidade da Amazônia (UNAMA) - Belém (PA), Brasil.

Endereço para correspondência: George Alberto da Silva Dias - Universidade do Estado do Pará (UEPA), Rua do Una, 156 - Telégrafo - CEP: 66050-540 - Belém (PA), Brasil - Email: georgealbertodias@yahoo.com.br

Fonte de financiamento: nenhuma.

Conflito de interesses: nada a declarar. 


\begin{abstract}
Introduction: Leprosy is an infectious disease that can lead to physical disability. The SALSA scale and the International Classification of Functioning, Disability and Health (ICF) may be useful in the clinical management of these patients. Thus, the objective was to correlate the ICF with the limitation of activity and restriction to participation for individuals with leprosy, using the SALSA scale. Methodology: This was an analytical and descriptive cross-sectional observational study, using the SALSA scale, related to mobility, self-care, work and skill domains. After the interview with the patients, the domains were codified by the ICF, using the codes related to activity and participation. Results: The sample consisted of 30 individuals, with a mean age of 38.4 , the majority being male. The SALSA scale mean score was 36.9 points, presenting a slight limitation of activities. The SALSA scale domains, together with the use of the ICF codes, show a slight to moderate difficulty in the great majority, with significant results ( $\mathrm{p} \leq 0.05$ ). The mobility domain was the only one to present task with complete difficulty. Conclusion: The SALSA scale showed a slight limitation of activity in patients with leprosy, but when related to ICF, there were larger limitations, especially in the mobility domain. Keywords: leprosy; International Classification of Functioning, disability and health; physical therapy.
\end{abstract}

\section{INTRODUÇÃO}

A hanseníase é uma disfunção crônica de origem infecciosa causada pelo Mycobacterium leprae. Possui amplo espectro clínico devido ao fato de ser uma doença granulomatosa descrita clinicamente de várias maneiras, podendo atingir pessoas de todas as idades e gêneros ${ }^{1}$. Em países em desenvolvimento, essa enfermidade apresenta-se como grave problema de saúde pública ${ }^{1}$. Sua transmissão ocorre em razão da inalação dos bacilos pelas vias aéreas superiores, e seu contágio depende do contato íntimo e prolongado com o indivíduo portador da doença, que não esteja em tratamento, podendo levar de 2 a 7 anos em um período de incubação $0^{2,3}$.

Segundo a Organização Mundial da Saúde (OMS), o número de casos novos de hanseníase no ano de 2014 foi de 213.899 em 121 países, mostrando ligeira redução em relação ao ano anterior, quando foram notificados 215.656 novos casos. No Brasil (o segundo país mais acometido), mesmo com a implantação do plano integrado de ações estratégicas para a eliminação da hanseníase, observou-se aumento desse número ${ }^{4}$, com 31.064 casos novos ${ }^{5}$. Nas regiões Norte, Centro-Oeste e Nordeste do país, a doença ainda apresenta alto coeficiente de surgimento de novos casos, e, no Estado do Pará, o percentual de detecção anual em crianças é de 1 a $13 \%$ (hiperendêmica) ${ }^{4,5}$.

A bactéria leva a alterações imunológicas que provocam manifestações características, como: alterações cutâneas, neurológicas, viscerais, oculares e otorrinolaringológicas. Destacam-se as lesões teciduais e desmielinizantes nos nervos periféricos dos membros superiores e inferiores, o que causa perda gradativa de sensibilidade cutânea e dores, mas que podem evoluir para atrofias, paresias e paralisias musculares, além de deformidades, promovendo o principal problema decorrente da hanseníase: a incapacidade física. Isso leva a uma redução substancial nas atividades de vida diárias de 57,4\% dos acometidos. Além disso, são mais susceptíveis a acidentes, queimaduras e amputações ${ }^{2,6-9}$.

Dessa forma, há uma significativa relação com a Classificação Internacional de Funcionalidade, Incapacidade e Saúde (CIF). A CIF é um dicionário de funcionalidade humana contendo itens que estão relacionados às partes do corpo e seu funcionamento, itens que tratam das atividades humanas, incluindo a participação social, e itens que influenciam nessas atividades, denominados fatores ambientais ${ }^{10}$.

A CIF permite o entendimento de que a doença é resultado de alterações da funcionalidade, e não a causadora. Além disso, proporciona a compreensão da situação diferenciada de incapacidade em pessoas com a mesma doença, porém inseridas em contextos diferentes. Esse é um importante fator, visto que o papel exercido pelo contexto é fundamental na sua análise, sendo, muitas vezes, o objeto de intervenção para melhoria do estado de funcionalidade ou para a prevenção da incapacidade ${ }^{10}$.

Diante disso, um instrumento de mensuração da limitação de atividade foi elaborado com base na CIF: a escala SALSA (Screening of Activity Limitation and Safety Awareness, ou Triagem de Limitação de Atividade e Consciência de Risco). O seu escore varia de 0 a 80 , em que valores altos são indicativos de crescente limitação. Os graus de limitação são classificados em: sem limitação (até 24), leve limitação (25 a 39), moderada limitação (40 a 49), grave limitação (50 a 59) e extrema limitação (60 a 80). É um instrumento validado no Brasil, com aplicação em diferentes cenários socioculturais, em pacientes que têm ou tiveram hanseníase e outras neuropatias periféricas ${ }^{11}$.

A fisioterapia pode atuar diretamente nessa disfunção em razão das incapacidades geradas. Isso é realizado buscando o retorno da funcionalidade por meio de exercícios terapêuticos que visam reduzir sintomas, como dor, edemas e limitações articulares. Ademais, orientações são feitas acerca da prevenção no que diz respeito à imobilização do membro afetado, marcha, utilização de órteses e alguns calçados ${ }^{6}$.

A prevenção possui um papel importante nessa temática, visto que sua principal função é evitar perdas funcionais. Além disso, inclui a identificação da instalação dessa patologia, levando a um tratamento precoce. Portanto, a educação em saúde torna-se um instrumento primordial nesse contexto, pois é por meio dela que a população será conscientizada ${ }^{7,12}$. Devido aos altos índices de sua prevalência, essa temática se torna relevante no âmbito da saúde comunitária4 . 
Ressalta-se, diante do exposto, que as incapacidades físicas causadas pela hanseníase são fatores importantes no que diz respeito à restrição dos indivíduos à participação social. Mesmo havendo cura e tratamento clínico para recuperação das incapacidades, avaliá-las é fundamental para evidenciar as repercussões motoras na vida cotidiana do indivíduo. Assim, o objetivo do presente estudo foi relacionar a CIF com a limitação de atividade e restrição à participação de indivíduos com hanseníase, aferidas por meio da escala SALSA.

\section{METODOLOGIA}

O estudo foi aprovado pelo Comitê de Ética em Pesquisa da Universidade da Amazônia (Parecer $\mathrm{n}^{\circ}$ 1.488.007), seguindo as normas da Resolução no 466/12 do Conselho Nacional de Saúde, relativa à pesquisa em seres humanos. Todos os participantes assinaram o Termo de Consentimento Livre e Esclarecido. Trata-se de um estudo observacional transversal analítico e descritivo.

\section{Amostra}

Participaram do estudo 30 indivíduos com hanseníase que frequentavam a Unidade de Referência Especializada em Dermatologia Sanitária Dr. Marcello Candia, em Marituba, no Pará. Os participantes foram selecionados por meio da amostragem não probabilística por conveniência e avaliados no período de abril a maio de 2016.

Foram incluídos indivíduos de ambos os sexos, maiores de 18 anos, com diagnóstico clínico de hanseníase, acompanhados no serviço de fisioterapia na unidade de referência e com algum grau de incapacidade identificado pelo serviço de triagem. Os critérios de exclusão foram: indivíduos portadores de outras neuropatias periféricas e de outras doenças dermatológicas, assim como pacientes que se encontrassem em estado de reação hansênica e alterações cognitivas.

\section{Procedimento de avaliação}

Foram coletadas informações dos prontuários sobre o exame físico dermatoneurológico padronizado e sistematizado para definição do escore Olho-Mão-Pé (Eye-Hand-Foot - EHF) e o grau máximo de incapacidade (GMI), além da ficha de avaliação própria da pesquisa contendo dados, como nome, sexo, idade atual, ocupação, tipo de hanseníase, uso de órteses, tempo de fisioterapia e orientação sobre prevenção de incapacidades. A coleta foi realizada em ambiente reservado por meio de uma entrevista. Também foi utilizada a escala SALSA, desenvolvida para medir a limitação de atividade e a consciência de risco em indivíduos acometidos por neuropatias periféricas, como o diabetes mellitus e a hanseníase ${ }^{13}$.

A escala SALSA é composta por 20 perguntas relacionadas a atividades diárias, que são separadas em quatro domínios: mobilidade (sentar ou agachar no chão; andar descalço; andar sobre chão irregular; andar distâncias longas), autocuidado (lavar o corpo todo; cortar as unhas das mãos e dos pés; segurar copo ou tigela com conteúdo quente), trabalho (trabalhar com ferramentas; carregar objetos ou sacolas pesadas; levantar objetos acima da cabeça; cozinhar; despejar líquidos quentes; abrir e fechar garrafas e vidros com tampa de rosca) e destreza (manipular objetos pequenos; usar botões; colocar linha na agulha; mexer com papel; apanhar coisas do chão), além de uma pergunta relacionada à acuidade visual ${ }^{13}$.

O escore final da escala SALSA varia de 10 a 80 pontos. Um escore baixo indica pouca dificuldade na realização de atividades diárias, enquanto que os escores mais altos revelam níveis crescentes de limitação de atividade. No escore da consciência de risco, o cálculo é realizado separadamente do escore SALSA, por meio da somatória de respostas sinalizadas com o desenho de um círculo (algumas atividades das colunas referentes às respostas, como "eu fisicamente não consigo" e "eu evito por causa do risco"). O escore de consciência de risco varia de 0 a 11, e os valores mais altos indicam consciência crescente dos riscos envolvidos em certas atividades ${ }^{13}$.

Para as variáveis avaliadas pela escala SALSA, apenas o domínio acuidade visual não foi codificado pela CIF, por se tratar do componente funções e estruturas do corpo, estando fora do objetivo proposto pela pesquisa. Desse modo, somente os itens mobilidade, autocuidado, trabalho e destreza foram codificados pela CIF (Tabela 1) por serem relacionados ao componente atividade e participação.

Os códigos da CIF só estão completos com a presença de pelo menos um qualificador, o qual indica a magnitude do nível de saúde. Assim, para os códigos utilizados nesta pesquisa, os qualificadores são descritos como: 0 não há problema; 1 para problema ligeiro; 2 para problema moderado; 3 para problema grave; 4 para problema completo. Todos os qualificadores foram utilizados para a aptidão de um indivíduo para executar uma tarefa ou uma ação, o que a CIF classifica como "capacidade".

\section{Análise estatística}

O software Microsoft Excel 2010 foi utilizado para entrada dos dados, bem como para a elaboração das tabelas. A análise estatística foi realizada por meio do software BioEstat 5.0, utilizando o Teste G (aderência) e Teste do Qui-quadrado, adotando nível alfa de significância de 5\%.

\section{RESULTADOS}

A amostra foi composta por 30 indivíduos com hanseníase, média e desvio-padrão de idade de $38,4 \pm 15,0$ anos, sendo a maioria do gênero masculino $(n=26,86,7 \% ; p=0,0001)$. O escore total de Olho-Mão-Pé(EHF) apresentou 11 indivíduos $(36,7 \%)$ com escores elevados, ou seja, apresentando deficiência. 
Tabela 1. Relação entre a escala SALSA e os códigos da CIF relacionados à atividade e participação

Escala SALSA

CIF

Mobilidade

Você se senta ou agacha no chão?

d4103 (sentar-se)

Você anda descalço?

d4509 (andar, não especificado)

Você anda sobre chão irregular?

d4502 (andar sobre superfícies diferentes)

Você anda distâncias mais longas?

d4501 (andar distâncias longas)

Autocuidado

Você lava seu corpo todo?

d5101 (lavar todo o corpo)

Você corta as unhas das mãos ou dos pés?

d5203 (cuidar das unhas das mãos)

Trabalho

Você trabalha com ferramentas?

Você carrega objetos ou sacolas pesadas?

d4401 (agarrar)

Você levanta objetos acima de sua cabeça?

d4301 (transportar nas mãos)

Você cozinha?

d4300 (levantar)

Você abre/fecha garrafas com tampa de rosca?

d6300 (preparar refeições simples)

Destreza

Você mexe/manipula objetos pequenos?

d4402 (manipular)

Você apanha pedaços de papel, mexe com papel/coloca papel em ordem? $\mathrm{d} 4305$ (pousar objetos)

Com relação ao grau de incapacidade, o grau $2(\mathrm{n}=18,60 \%)$ foi o predominante, indicando a presença de incapacidades e deformidades com resultados estatisticamente significantes $(\mathrm{p}=0,0007)$ (Tabela 2).

O escore SALSA variou de 21 a 53 pontos, com pontuações bem distribuídas entre os pacientes. A pontuação média da escala foi de $36,9 \pm 8,3$ pontos, em que a grande maioria evidenciou leve limitação das atividades. Escores acima de 50 pontos foram alcançados somente por um paciente (3,3\%), indicando grave dificuldade na realização das atividades diárias. Para o escore de consciência de risco (escala de 0 a 11), houve variação de 0 a 7: 15 indivíduos (50\%) pontuaram 0; 6 indivíduos (20\%) pontuaram 1; 6 indivíduos (20\%) pontuaram 2; 2 indivíduos $(6,7 \%)$ pontuaram $6 ; 1$ indivíduo $(3,3 \%)$ pontuou 7 , conforme mostra a Tabela 3, indicando baixa percepção na consciência de risco. Esses resultados mostram que, mesmo apresentando graus elevados de incapacidade (EHF e GMI), os pacientes possuem baixa consciência de risco, conforme mostrado pela escala SALSA.

Para o uso da CIF, foram utilizados quatro códigos para mobilidade, dois para autocuidado, cinco para trabalho e dois para destreza. No domínio mobilidade, a grande maioria apresentou dificuldade leve a moderada. Para "sentar-se", 17 pacientes (56,7\%, $\mathrm{p}=0,0002)$ tiveram dificuldade moderada; para "andar sobre superfícies diferentes", 15 pacientes $(50 \%, \mathrm{p}=0,13)$ apresentaram dificuldade leve; para "andar distâncias longas", 14 pacientes $(46,7 \%, \mathrm{p}=0,02)$ demonstraram dificuldade leve. Porém, para "andar, não especificado", 16 pacientes $(53,3 \%, \mathrm{p}=0,03)$ tiveram dificuldade completa, com resultados significantes (Tabela 4).

Para o domínio autocuidado, as dificuldades encontradas foram leves, ou seja, 22 pacientes $(73,3 \%, \mathrm{p}=0,01)$ apresentaram dificuldade para "lavar todo o corpo", e 14 pacientes $(46,7 \%$, $\mathrm{p}=0,04)$, para "cuidar das unhas das mãos". Para o domínio trabalho, foi observada predominância de dificuldade leve a
Tabela 2. Caracterização (número absoluto e percentual) demográfica, EHF e GMI da população do estudo

\begin{tabular}{lccc}
$\quad$ Variáveis & N & $\%$ & p-valor \\
Idade & & $38,4 \pm 15,0$ anos & \\
Sexo & & & \\
$\quad \begin{array}{l}\text { Masculino } \\
\text { Feminino }\end{array}$ & 26 & $86,7 \%$ & $\mathrm{p}=0,0001^{*}$ \\
Escore EHF & 4 & $13,3 \%$ & \\
0 & & & \\
1 & 2 & $6,7 \%$ & \\
2 & - & - & \\
3 & 9 & $30 \%$ & \\
4 & 4 & $13,3 \%$ & \\
5 & 4 & $13,3 \%$ & \\
6 & - & - & \\
7 & 8 & $26,7 \%$ & \\
8 & - & - & \\
GMI & 3 & $10 \%$ & \\
0 & & & \\
1 & 2 & $6,7 \%$ & \\
2 & 10 & $60 \%$ & \\
\hline
\end{tabular}

EHF: Eye, Hand, Foot (escore total de olho, mão e pé); GMI: grau máximo de incapacidade; ${ }^{*}$ Teste do Qui-quadrado $(\mathrm{p} \leq 0,05) ;{ }^{* *}$ Teste $\mathrm{G}$ (aderência) $(\mathrm{p} \leq 0,05)$

Tabela 3. Escore (número absoluto e percentual) SALSA da população do estudo

\begin{tabular}{ccc}
\multicolumn{1}{c}{ Variáveis } & $\mathbf{N}$ & $\%$ \\
Salsa & $36,9 \pm 8,3$ & \\
CR & & \\
0 & 15 & $50 \%$ \\
1 & 6 & $20 \%$ \\
2 & 6 & - \\
3 & - & - \\
4 & - & - \\
5 & - & $6,7 \%$ \\
6 & 2 & $3,3 \%$ \\
7 & 1 & - \\
8 & - & - \\
9 & - & - \\
10 & - & - \\
11 & - &
\end{tabular}

CR: consciência de risco; (-) Dado numérico igual a zero 
Tabela 4. Restrição à participação social de pacientes com hanseníase por meio do uso dos códigos da CIF

\begin{tabular}{|c|c|c|c|c|c|}
\hline \multirow{3}{*}{ VARIÁVEIS } & \multicolumn{4}{|c|}{ QUALIFICADORES DA CIF } & \multirow{3}{*}{ p-valor } \\
\hline & 1 & 2 & 3 & 4 & \\
\hline & $\mathrm{n}(\%)$ & $\mathbf{n}(\%)$ & $\mathbf{n}(\%)$ & $\mathrm{n}(\%)$ & \\
\hline \multicolumn{6}{|l|}{ MOBILIDADE } \\
\hline d4103 (sentar-se) & $12(40 \%)$ & $17(56,7 \%)$ & $1(3,3 \%)$ & - & $0,0002^{\star}$ \\
\hline d4509 (andar, não especificado) & $3(10 \%)$ & $4(13,3 \%)$ & $7(23,3 \%)$ & $16(53,3 \%)$ & $0,03^{*}$ \\
\hline d4502 (andar sobre superfícies diferentes) & $15(50 \%)$ & $9(30 \%)$ & $6(20 \%)$ & - & 0,13 \\
\hline d4501 (andar distâncias longas) & $14(46,7 \%)$ & $8(26,7 \%)$ & $7(23,3 \%)$ & $1(3,3 \%)$ & $0,02^{*}$ \\
\hline \multicolumn{6}{|l|}{ AUTOCUIDADOS } \\
\hline d5101 (lavar todo o corpo) & $22(73,3 \%)$ & $8(26,7 \%)$ & - & - & $0,01^{\star}$ \\
\hline d5203 (cuidar das unhas das mãos) & $14(46,7 \%)$ & $12(40 \%)$ & $4(13,3 \%)$ & - & $0,04^{*}$ \\
\hline \multicolumn{6}{|l|}{ TRABALHO } \\
\hline d4401 (agarrar) & $9(30 \%)$ & $15(50 \%)$ & $5(16,7 \%)$ & $1(3,3 \%)$ & $0,008^{*}$ \\
\hline d4301 (transportar nas mãos) & $3(10 \%)$ & $12(40 \%)$ & $8(26,7 \%)$ & $7(23,3 \%)$ & 0,58 \\
\hline d4300 (levantar) & $19(63,3 \%)$ & $11(36,7 \%)$ & - & - & 0,19 \\
\hline d6300 (preparar refeições simples) & $12(40 \%)$ & $7(23,3 \%)$ & $4(13,3 \%)$ & $7(23,3 \%)$ & 0,93 \\
\hline d4453 (rodar ou torcer as mãos ou os braços) & $8(26,7 \%)$ & $20(66,7 \%)$ & $2(6,7 \%)$ & - & $0,0002^{*}$ \\
\hline \multicolumn{6}{|l|}{ DESTREZA } \\
\hline d4402 (manipular) & $8(26,7 \%)$ & $17(56,7 \%)$ & $5(16,7 \%)$ & - & $0,02^{*}$ \\
\hline d4305 (pousar objetos) & $14(46,7 \%)$ & $14(46,7 \%)$ & $1(3,3 \%)$ & $1(3,3 \%)$ & $<0,0001^{\star}$ \\
\hline
\end{tabular}

(-) Dado numérico igual a zero; CIF: Classificação Internacional de Funcionalidade, Incapacidade e Saúde; Qualificadores: (1) dificuldade ligeira; (2) dificuldade moderada; (3) dificuldade grave; (4) dificuldade completa; ${ }^{\star}$ Teste $\mathrm{G}$ (aderência) $(\mathrm{p} \leq 0,05)$

moderada, sendo 15 indivíduos $(50 \%, \mathrm{p}=0,008)$ com dificuldade moderada para "agarrar" e 20 (66,7\%, p=0,0002) para "rodar ou torcer as mãos ou os braços", com resultados significantes. Mesmo não apresentando resultados relevantes, foi possível observar que 12 indivíduos $(40 \%, \mathrm{p}=0,58)$ apresentaram dificuldade moderada para "transportar nas mãos", 19 indivíduos (63,3\%, $\mathrm{p}=0,19)$ tiveram dificuldade leve para "levantar" e 12 indivíduos $(40 \%, \mathrm{p}=0,93)$ demonstraram dificuldade leve para "preparar refeições simples" (Tabela 4).

Para o domínio destreza, foi possível constatar que as dificuldades encontradas foram consideradas leves e moderadas, apresentando resultados estatisticamente significantes: 17 pacientes $(56,7 \%, \mathrm{p}=0,02)$ apresentaram dificuldade moderada para "manipular" e 14 pacientes $(46,7 \%, \mathrm{p}<0,0001)$ tiveram tanto dificuldade leve quanto moderada para "pousar objetos". Assim, foi observado que o domínio mobilidade apresentou codificação com dificuldade completa, diferentemente do observado nos outros domínios (Tabela 4).

\section{DISCUSSÃO}

Pesquisas realizadas em Sobral, no Estado do Ceará, de 1997 a 2003, mostram que a maioria da população naquela região (70\%) adquiriu a forma paucibacilar, isto é, a versão leve da doença, com manchas na pele e sem incapacidades físicas. Porém, nos anos seguintes, aumentou-se o número de casos da forma multibacilar, alcançando $62 \%$ da população hansênica em 2002 e quase $86 \%$ em $2003^{14}$, o que corrobora a amostra do presente estudo, no qual todos os voluntários possuíam a forma multibacilar. Também observou-se que a maioria da amostra era masculina, fortalecendo dados nacionais e de outros estudos ${ }^{8,15}$.

Os dados mostraram que os pacientes avaliados neste estudo apresentaram incapacidades físicas consideradas graves, porém tiveram baixa consciência de risco. Esse dado corrobora outro estudo ${ }^{16}$ que avaliou 69 pacientes com hanseníase após alta, utilizando a escala SALSA, e também verificou que os pacientes possuíam baixa percepção de risco. Em outras palavras, há necessidade de maior orientação sobre o domínio afetado no indivíduo, uma vez que é preciso orientar o paciente quanto à consciência do risco de incapacidades físicas, o que pode evitar, assim, maiores limitações de atividades diárias.

Destaca-se que o domínio autocuidado, apesar de ter apontado dificuldades consideradas leves, na tarefa "lavar todo o corpo (d5101)", apresentou o maior número de pacientes prejudicados, um total de 22 pacientes $(73,3 \%, \mathrm{p}=0,01)$. Sabe-se que, ao tratar de barreiras funcionais, as mais frequentes envolvem as atividades básicas de vida diária (ABVDs) (80\%), como a higiene pessoal e a locomoção ${ }^{17}$ - tarefas também encontradas como prejudicadas nos resultados da presente pesquisa. Concomitantemente estudos ${ }^{17,18}$ afirmam ter encontrado queixas na execução de tarefas de higiene corporal. Outro estudo $^{18}$ destaca também a higiene bucal prejudicada pelas alterações nos membros superiores.

Além disso, é importante ressaltar o domínio mobilidade, que apresentou dificuldades consideradas completas, visto que é um fato bastante comum para os indivíduos que possuem 
essa doença por causa das alterações provocadas nos membros. Dados apontam ${ }^{9,19}$ que o maior número de incapacidades está relacionado à perda de sensibilidade e ao aparecimento de lesões nos membros, o que ocasiona certa dependência e insegurança, prejudicando, assim, a mobilidade do indivíduo.

Por meio da avaliação da escala SALSA, o déficit em pacientes multibacilares ocorreu nos membros superiores e, em alguns casos, nos membros inferiores, causando dificuldades em deambular e manter o centro de apoio em atividades como sentar ou levantar. Isso corrobora outro estudo ${ }^{2}$, o qual destaca o comprometimento nessas mesmas áreas e afirma que, geralmente, essas lesões são responsáveis por causar incapacidades.

Outra pesquisa ${ }^{6}$, que utilizou cinco portadores de hanseníase, de ambos os sexos, com neurites crônicas de membros superiores, encontrou comprometimento do nervo ulnar em $70 \%$ e no nervo ulnar e mediano em 30\%. Trata-se de um importante achado, visto que a integridade desse sistema controla a força imprimida, portanto seu comprometimento leva à redução da preensão palmar.

Também outro estudo ${ }^{7}$ encontrou resultados semelhantes ao analisar 26 pacientes de ambos os sexos. Quando os membros superiores foram avaliados, cinco apresentaram mãos com garras móveis, duas, com hipotrofia, e nove casos, ressecamento da pele. Sobre os membros inferiores, encontraram-se duas úlceras plantares, uma deformidade em pé equino e 20 casos de ressecamento da pele. Isso mostra a significância encontrada entre esses achados e as formas multibacilares da doença, por meio da mesma escala usada no presente estudo ${ }^{8}$.

A escala SALSA vem ganhando espaço nas pesquisas e chamando atenção não só para as limitações físicas, mas também para a funcionalidade do indivíduo. Nesse sentido, o deslocamento dos voluntários para as unidades de tratamento é considerado uma barreira porque algumas delas se encontram em locais distantes. Esse fato é agravado pelas poucas unidades especializadas, fazendo-se necessária uma ampliação para setores de fácil acesso aos pacientes. Além disso, devem-se enriquecer profissionais da área quanto ao conhecimento da doença, os quais, sob a visão dos acometidos, ainda precisam avançar muito nessa questão.

É notório que a assistência básica à saúde não deve estagnar, mas sim expandir-se, investindo mais nas estratégias de prevenção e de conscientização da população sobre a hanseníase, principalmente porque as regiões Norte, Nordeste e Centro-Oeste ainda apresentam grau de acometimento alto e porque estudos descritos ${ }^{14,16,20}$ evidenciam que a incapacidade física é crescente.

Diretamente associado ao aumento das incapacidades, está a dificuldade de diagnóstico precoce, por causa da predominância das formas passivas de detecção em virtude da busca ativa de casos. O modelo de atenção à saúde praticado pelos serviços, contrariando a Política Nacional de Atenção Básica, valoriza o atendimento individual, deixando de lado as ações coletivas e em grupo, atrasando a principal ferramenta de redução das complicações da doença, que seria possibilitar a intervenção imediata com o diagnóstico precoce ${ }^{9}$.

Em contrapartida, a avaliação das incapacidades precocemente possibilita a prevenção e, até mesmo, a reversão das sequelas, pois atenta-se para o paciente que apresenta comprometimento nervoso, por possuir maior risco de desenvolver incapacidades. Diante disso, os programas de controle devem ser mais criteriosos na avaliação inicial.

A fisioterapia pode contribuir para o tratamento dessa disfunção, melhorando a capacidade funcional e, consequentemente, a qualidade de vida desses indivíduos. Pode também proporcionar melhora da força e do trofismo muscular por meio de exercícios terapêuticos ativos resistidos, os quais podem ser realizados com uso de materiais simples, como bolas, faixas e ligas elásticas, lenços, além de exercícios que envolvam resistência manual, como sugere o Manual de Prevenção de Incapacidades do Ministério da Saúde ${ }^{12}$. Ainda, pode trazer flexibilidade por meio de alongamento muscular passivos ou ativos e diminuição da dor com mobilização neural ${ }^{6,21}$.

Esse processo deve estar aliado ao tratamento farmacológico e à assistência multiprofissional, para então obter uma recuperação mais eficaz. Porém, para minimização das sequelas, déficits funcionais e cura eficaz, é necessário que o tratamento seja respeitado pelos pacientes.

\section{CONCLUSÃO}

Pode-se concluir que a escala SALSA mostrou que os pacientes apresentavam leve limitação das atividades, porém, quando relacionado com a CIF, os dados apresentaram variação leve a moderada na maioria dos casos. O único domínio que apresentou codificação com dificuldade completa foi o domínio mobilidade para a tarefa "andar, não especificado".

A utilização da CIF daria subsídio para identificar, de fato, qual domínio é o mais acometido pelo paciente por meio de uma avaliação mais detalhada, uma vez que a avaliação não se detém em uma somatória de itens, resultando em um escore final, como acontece na escala SALSA. Assim, o uso da CIF seria capaz de orientar em relação ao domínio específico a ser abordado, principalmente mãos ou pés.

Ainda é um grande desafio a utilização da CIF pelos profissionais de saúde que atuam rotineiramente com indivíduos com hanseníase. Este estudo mostra a importância de utilização de ferramentas para garantir a integralidade da atenção ao paciente com hanseníase em programas de prevenção de incapacidade ou até mesmo na reabilitação, garantindo intervenções eficientes. 


\section{REFERÊNCIAS}

1. Gonçalves A. Realities of leprosy control: updating scenarios. Rev Bras Epidemiol. 2013;16(3):611-21. PMid:24896275. http://dx.doi.org/10.1590/ S1415-790X2013000300006.

2. Brasil. Ministério da Saúde. Hanseníase: informações para agentes comunitários de saúde. Brasília: Secretaria de Políticas de Saúde; 2001.

3. Lastória JC, Abreu MAMM. Leprosy: review of the epidemiological, clinical, and etiopathogenic aspects. Part 1. An Bras Dermatol. 2014;89(2):205-18. PMid:24770495.

4. Brasil. Ministério da Saúde. Plano integrado de ações estratégicas de eliminação da hanseníase, filariose, esquistossomose e oncocercose como problema de saúde pública, tracoma como causa de cegueira e controle das geohelmintíases: plano de ação 2011-2015. Brasília: Ministério da Saúde; 2013.

5. World Health Organization. Relevé épidémiologique hebdomadaire. Wkly Epidemiol Rec. 2015;90(36):461-76. PMid:26343055.

6. Lima GM, Miranda MGR, Ferreira TCR. Ação do exercício terapêutico nas neurites crônicas de membros superiores em pacientes portadores de hanseníase atendidos na Unidade de Referência Especializada em Dermatologia Sanitária Dr. Marcello Candia. Hansen Int. 2009;34(1):916.

7. Rodini FCB, Gonçalves M, Barros ARSB, Mazzer N, Elui VMC, Fonseca MCR. Prevenção de incapacidade na hanseníase com apoio em um manual de autocuidado para pacientes. Fisioterapia e Pesquisa. 2010;17(2):157-66. http://dx.doi.org/10.1590/S1809-29502010000200012.

8. Ikehara E, Nardi SMT, Ferrigno ISV, Pedro HSP, Paschoal VD. Escala salsa e grau de incapacidades da Organização Mundial de Saúde: avaliação da limitação de atividades e deficiência na hanseníase. Acta Fisiatr. 2010;17(4):169-74.

9. Ribeiro GC, Lana FCF. Incapacidades físicas em hanseníase: caracterização, fatores relacionados e evolução. Cogitare Enferm. 2015;20(3):496-503. http://dx.doi.org/10.5380/ce.v20i3.41246.

10. Araujo ES. CIF: uma discussão sobre linearidade no modelo biopsicossocial. Rev Fisioter S Fun. 2013;2(1):6-13.

11. SALSA Collaborative Study Group. SALSA scale (Screening Activity Limitation and Safety Awareness): users manual. Version 1. Netherlands; 2010.
12. Brasil. Ministério da Saúde. Manual de prevenção de incapacidades. Brasília: Ministério da Saúde; 2008. (Cadernos de Prevenção e Reabilitação em Hanseníase).

13. Ebenso J, Fuzikawa P, Melchior H, Wexler R, Piefer A, Min CS, et al. The development of a short questionnaire for screening of activity limitation and safety awareness (SALSA) in clients affected by leprosy or diabetes. Disabil Rehabil. 2007;29(9):689-700. PMid:17453991. http://dx.doi. org/10.1080/09638280600926587.

14. Campos SSL, Ramos Jr NA, Kerr-Pontes LRS, Heukelbach J. Epidemiologia da hanseníase no Município de Sobral, Estado do Ceará-Brasil, no Período de 1997 a 2003. Hansenol Int. 2005;30(2):167-73.

15. Brasil. Ministério da Saúde. Vigilância em Saúde: situação epidemiológica da hanseníase no Brasil. Brasília: Ministério da Saúde; 2008.

16. Barbosa JC, Ramos Jr AN, Alencar MJF, Castro CGJ. Pós-alta em Hanseníase no Ceará: limitação da atividade funcional, consciência de risco e participação social. Rev Bras Enferm. 2008;61:727-33. PMid:19009115. http://dx.doi. org/10.1590/S0034-71672008000700012.

17. Beltrame RT, Marciano LHSC, Fonseca MS, Prado RBR. Estratégias de enfrentamento utilizadas pelo paciente com deficiências físicas na hanseníase. Mimesis. 2015;36(1):117-38.

18. Filgueira AA, Paresque MAC, Carneiro SMF, Teixeira AKM. Saúde bucal em indivíduos com hanseníase no município de Sobral, Ceará. Epidemiol Serv Saúde. 2014;23(1):155-64. http://dx.doi.org/10.5123/ S1679-49742014000100015.

19. Rocha AKAA, Silva ED Junior, Novaes MM, Franco CIF. Análise da independência funcional em pacientes com neuropatia hanseniana assistidos pelo centro de referência em hanseníase da cidade de Campina Grande-Paraíba. Rev Saúde e Biol. 2014;9(3):8-16.

20. Cunha ACSR. Censo de deficiências e incapacidades físicas por hanseníase e monitoramento pela escala salsa do plano de autocuidado para prevenção de incapacidades de pacientes atendidos em um centro de referência nacional do Brasil [dissertação]. Uberlândia: Universidade Federal de Uberlândia; 2012.

21. Ferreira JL, Cerdeira DQ, Nunes TT, Guimarães DF, Liberato FR. Atuação da fisioterapia no acompanhamento de pacientes com hanseníase. Fisioter Bras. 2016;17(5):472-9.
Recebido em: Nov. 02, 2016 Aprovado em: Ago. 04, 2017 